\title{
Interaction of Solid Lipid Nanoparticles and Specific Proteins of the Corona Studied by Surface Plasmon Resonance
}

\author{
Mauricio E. Di Ianni, ${ }^{1}$ Germán A. Islan, ${ }^{2}$ Cecilia Y. Chain, ${ }^{3}$ Guillermo R. Castro, ${ }^{2}$ \\ Alan Talevi, ${ }^{1}$ and María E. Vela ${ }^{3}$ \\ ${ }^{1}$ Laboratorio de Investigación y Desarrollo de Bioactivos (LIDeB), Departamento de Ciencias Biológicas, \\ Facultad de Ciencias Exactas, Universidad Nacional de La Plata, La Plata, Argentina \\ ${ }^{2}$ Laboratorio de Nanobiomateriales, Centro de Investigación y Desarrollo de Fermentaciones Industriales (CINDEFI), \\ UNLP-CONICET, CCT La Plata, Departamento de Química, Facultad de Ciencias Exactas, Universidad Nacional de La Plata, \\ Calle 47 y 115, La Plata, Argentina \\ ${ }^{3}$ Instituto de Investigaciones Fisicoquímicas Teóricas y Aplicadas (INIFTA), CONICET-UNLP, CC 16 Suc 4, La Plata, \\ 1900 Buenos Aires, Argentina
}

Correspondence should be addressed to Cecilia Y. Chain; yamil.chain@gmail.com

Received 30 March 2017; Revised 5 June 2017; Accepted 15 June 2017; Published 25 July 2017

Academic Editor: Kimberly Hamad-Schifferli

Copyright (C) 2017 Mauricio E. Di Ianni et al. This is an open access article distributed under the Creative Commons Attribution License, which permits unrestricted use, distribution, and reproduction in any medium, provided the original work is properly cited.

The applications of pharmaceutical and medical nanosystems are among the most intensively investigated fields in nanotechnology. A relevant point to be considered in the design and development of nanovehicles intended for medical use is the formation of the "protein corona" around the nanoparticle, that is, a complex biomolecular layer formed when the nanovehicle is exposed to biological fluids. The chemical nature of the protein corona determines the biological identity of the nanoparticle and influences, among others, the recognition of the nanocarrier by the mononuclear phagocytic system and, thus, its clearance from the blood. Recent works suggest that Surface Plasmon Resonance (SPR), extensively employed for the analysis of biomolecular interactions, can shed light on the formation of the protein corona and its interaction with the surroundings. The synthesis and characterization of solid lipid nanoparticles (SLN) coated with polymers of different chemical nature (e.g., polyvinyl alcohol, chitosans) are reported. The proof-of-concept for the use of SPR technique in characterizing protein-nanoparticle interactions of surface-immobilized proteins (immunoglobulin G and bovine serum albumin, both involved in the formation of the corona) subjected to flowing SLN is demonstrated for non-chitosan-coated nanoparticles. All assayed nanosystems show more preference for IgG than for BSA, such preference being more pronounced in the case of polyvinyl-alcohol-coated SLN.

\section{Introduction}

Pharmaceutical and medical applications of nanosystems represent a growing area in the flourishing field of nanotechnology, including developments in advanced pharmaceutical nanocarriers and nanodiagnostics [1-3]. Nanosized carriers of very diverse composition are being actively investigated, including metallic, polymeric, ceramic, and lipidic nanoparticles and also hybrid systems by combining such materials $[4,5]$.

One of the main limitations of these kinds of delivery systems is their short circulation time due to the opsonization phenomenon and subsequent uptake by the mononuclear phagocytic system (MPS). After exposure of nanocarriers to biological fluids, biomolecules are rapidly adsorbed onto their surface forming the protein corona. The corona composition determines the biological identity of the nanoparticle and influences its toxicity, biological fate, circulation time, cell uptake, and drug release kinetics [6, 7]. At first, the protein corona includes considerable amounts of low-affinity proteins which abound in the biological medium. Over time, the composition of the corona is enriched with high affinity proteins [7]. Interestingly, some of the adsorbed proteins are opsonins which promote the uptake and degradation of the 
nanoparticles by the cells of the mononuclear phagocytic system (MPS) (mainly located in the liver, spleen, and lymph nodes). Among the proteins commonly found in the corona, IgG and proteins involved in the complement pathway typically act as opsonins (promoting uptake by MPS) whereas serum albumin and apolipoproteins enhance circulation time [8]. The clearance of the nanoparticles by the MPS reduces the bioavailability of the encapsulated drug $[9,10]$. A considerable amount of evidence, however, indicates that this phenomenon can be reduced by coating the nanoparticles with different polymeric substances [11-13].

Lipid-based nanosystems stand out because of their nontoxic, biocompatible and environmentally friendly composition. In fact, most of the pharmaceutical nanosystems that have received approval from national health authorities and are already in use in the clinics are of lipidic nature (liposomes) [14]. Solid lipid nanoparticles (SLN), consisting in a solid lipid core matrix stabilized by surfactants, are of particular interest to encapsulate lipophilic drugs [15]. SLN are not only versatile with respect to their composition, but it is also possible to regulate both their particle size and surface characteristics [15]. They are relatively easy to prepare with "green" procedures, resulting in a final product that displays good physicochemical stability compatible with long-term storage, lyophilization, and sterilization processes [16, 17].

Surface Plasmon Resonance (SPR) is a label-free detection method which is particularly suitable for biomolecular interactions studies, allowing high sensitivity measurements in real time. This optical technique is based on the changes that occur in the refractive index due to modifications in the chemical composition in the proximity of thin metal layers (typically gold and silver) that constitute the sensor chips of the SPR equipment [18-21]. The study of proteinnanoparticles interactions by SPR can be performed in two ways; one entails the flow of proteins onto NP immobilized on the SPR sensor surface and the other involves the immobilization of the protein and the injection of nanoparticles throughout the fluidic system. A higher sensitivity is expected for the latter option due to the higher mass of the NP providing that the binding of the proteins to the sensor surface does not alter their typical configuration regarding protein/NP interactions. Previous SPR studies on the interactions of nanovehicles with proteins of the bloodstream include the investigation of the corona formation by employing serum or plasma $[22,23]$ and the interaction of nanoparticles with individual proteins known to adsorb onto the surface of nanocarriers [24], an approach that has been considered for the present experimental design.

In this work the synthesis of SLN by ultrasonication of cetyl esters mixture in a dispersant agent (Poloxamer 188) and, in some cases, polyvinyl alcohol or chitosan as chemical coatings is reported. The particle size, zeta potential, and polydispersity index of the obtained SLN solutions are characterized by Dynamic Light Scattering- (DLS-) based measurements. Morphology and size distribution are corroborated by Transmission Electron Microcopy (TEM). The nanoparticle size in the presence of serum is also characterized by DLS. SPR is then used to monitor proteinnanoparticle interactions involved in the formation of the corona, by immobilizing selected proteins of the corona in the sensor surface that is subjected to the flow of SLN solutions. As a proof-of-principle, two proteins of the corona with opposite effects on the circulation time in the bloodstream are chosen for the SPR experiments: IgG (that promotes the clearance of the nanovehicle by the MPS) and BSA (that enhances circulation time).

\section{Experimental}

2.1. Reagents and Materials. The cetyl esters mixture Crodamol $^{\mathrm{TM}}$ SS (melting point $\sim 44^{\circ} \mathrm{C}$ ) was kindly donated by Croda (Argentina). Pluronic ${ }^{\circledR}$ F68, polyvinyl alcohol (PVA, mol wt $=13-23 \mathrm{kDa}, 98.0$ hydrolyzed), low and medium molecular weights chitosans $(50-190 \mathrm{kDa}$ and $190-310 \mathrm{kDa}$, resp., 75-85\% deacetylated), Bovine Serum Albumin (BSA, $\mathrm{MW}=66.5 \mathrm{kDa}$.$) , 11-mercaptoundecanoic acid (MUA),$ $\mathrm{N}$-hydroxysuccinimide (NHS), ethanolamine hydrochloride, and $\mathrm{N}$-(3-dimethylaminopropyl)- $\mathrm{N}^{\prime}$-ethylcarbodiimide hydrochloride (EDC) were purchased from Sigma-Aldrich (St. Louis, Mo, USA). Potassium chloride, sodium chloride, sodium dihydrogen phosphate, and disodium hydrogen phosphate were obtained from J. T. Baker (Pasadena, Ca, USA). Sodium carbonate was purchased from Biopack (Buenos Aires, Argentina). Commercial gold substrates (SPR102-AU) were obtained from BioNavis ${ }^{\mathrm{TM}}$ (Tampere, Finland). The IgG antibody used in the experiments is monoclonal IgG1 anti-DNP 112D5 [25] and it was kindly provided by Drs. Guillermo Docena and Martin Rumbo from IIFP (CONICET-UNLP), La Plata (Argentina).

2.2. Synthesis of Solid Lipid Nanoparticles. SLN were prepared by the ultrasonication method [26]. Briefly, $400 \mathrm{mg}$ of lipid $(2.0 \% \mathrm{w} / \mathrm{v})$ was melted in a water bath at $70^{\circ} \mathrm{C}$. Later, hot aqueous solution $\left(70^{\circ} \mathrm{C}, 20 \mathrm{ml}\right)$ containing $3 \%(\mathrm{w} / \mathrm{v})$ of Pluronic F68 was added to the lipid phase. Immediately, the mixture was ultrasonicated for $30 \mathrm{~min}$ (50\% amplitude) using an ultrasonic processor ( $130 \mathrm{~W}$, Cole-Parmer, USA) equipped with a $3 \mathrm{~mm}$ titanium tip. Then, the dispersion was cooled down at room temperature and stored at $5^{\circ} \mathrm{C}$ $[27,28]$. The obtained SLN formulation is denoted, from here, P188. The same synthesis, but using $4.5 \%(\mathrm{w} / \mathrm{v})$ of Pluronic F68, yielded a SLN formulation called P188+. Three SLN formulations including coating polymers were prepared, by adding polyvinyl alcohol, low molecular weight chitosan, and medium molecular weight chitosan to the aqueous phase (called PVA, CL, and CM, resp.). In the case of SLN formulations $\mathrm{CL}$ and $\mathrm{CM}$, the procedure involved the dissolution of $2.0 \%(\mathrm{w} / \mathrm{v})$ chitosan in $0.1 \%$ acetic acid at $\mathrm{pH} 4.0$ and the preheating to $70^{\circ} \mathrm{C}$ before the addition of the polymer to the aqueous phase. The compositions of all formulations are shown in Table 1.

2.3. Particle Size, Zeta Potential ( $\zeta)$, and Polydispersity Index $(P D I)$. The mean diameter and size distribution were measured by photon correlation spectroscopy (PCS) (Nano ZS Zetasizer, Malvern Instruments Corp, UK) at $25^{\circ} \mathrm{C}$ in polystyrene cuvettes with a path length of $10 \mathrm{~mm}$. The zeta potential was determined by laser Doppler anemometry also 
TABLE 1: Composition of the aqueous phase of SLN formulations*. In all cases, the lipid used was Crodamol SS.

\begin{tabular}{lcc}
\hline Formulation $^{*}$ & Aqueous phase components & Composition (\%) \\
\hline SLN P188 & Pluronic F68 & 3 \\
SLN PVA & Pluronic F68: PVA & $2.5: 0.5$ \\
SLN CM & Pluronic F68 : MMWC & $2.5: 0.5$ \\
SLN CL & Pluronic F68: LMWC & $2.5: 0.5$ \\
SLN P188+ & Pluronic F68 & 4.5 \\
\hline
\end{tabular}

using the Nano ZS Zetasizer. Measurements were performed in capillary cells with path lengths of $10 \mathrm{~mm}$, using deionized water obtained from a Milli-Q system and phosphate buffered saline (PBS) as diluting media. Additional measurements were accomplished in SLN formulations preincubated in Fetal Bovine Serum (FBS) during one hour [29]. In all cases the measurements were performed in triplicate.

2.4. Transmission Electron Microscopy (TEM). The nanoparticle dispersion was ten times diluted with ultrapure water and a drop of the dispersion was spread onto a collodioncoated $\mathrm{Cu}$ grid (400 mesh). Liquid excess was drained with filter paper. One drop of phosphotungstic acid was added to the dispersion, for contrast enhancement. Finally, TEM analysis was performed using Jeol-1200 EX II-TEM microscope (Jeol, MA, USA.).

2.5. Preparation of the Sensor Surfaces. Single thiol SAMs were obtained by overnight incubation of the gold substrates in $50 \mu \mathrm{M}$ MUA ethanolic solution at room temperature. The obtained surfaces were washed with Milli-Q ${ }^{\circledR}$ water and dried in a stream of nitrogen before SPR measurements.

2.6. SPR Measurements. Measurements were performed with a SPR Navi ${ }^{\mathrm{TM}} 210 \mathrm{~A}$ (BioNavis) instrument. The setup is equipped with two incident laser wavelengths, $670 \mathrm{~nm}$ and $785 \mathrm{~nm}$, two independent flow channels, and inlet tubing and outlet (waste) tubing. In this work, both of the flow channels were measured in parallel with $670 \mathrm{~nm}$ incident light. The measurement temperature was kept constant at $22^{\circ} \mathrm{C}$, and the flow rate used for protein immobilization and for monitoring the SLN interactions with the immobilized proteins was $10 \mu \mathrm{L} / \mathrm{min}$.

The working mode was "in parallel," by immobilizing IgG in one flow cell and BSA in the other. The immobilization of the proteins to the single SAMs was performed by the wellknown carbodiimide coupling reaction [30]. The first step is the activation of the MUA-carboxyl groups on the surface with a mixture of $0.1 \mathrm{M} \mathrm{EDC}$ and $0.1 \mathrm{M}$ NHS to give reactive succinimide esters. IgG $50 \mu \mathrm{g} / \mathrm{mL}$ in $10 \mathrm{mM}$ sodium acetate $\mathrm{pH} 4.6$ and BSA $100 \mu \mathrm{g} / \mathrm{mL}$ in $10 \mathrm{mM}$ sodium acetate $\mathrm{pH}$ 4 are then passed through flow cells 1 and 2, respectively, over the surface, and the esters react spontaneously with primary amine groups of the proteins. The immobilization process concludes by blocking remaining succinimide esters using $1 \mathrm{M}$ ethanolamine, $\mathrm{pH}$ 8.5. A simplified scheme of the sensor surface is shown in Figure 1. The same schedule of carbodiimide coupling reaction, but flowing buffer solution instead of protein solution, was performed to obtain control surfaces.

SLN solutions $(2 \mathrm{mg} / \mathrm{ml}$ in PBS $\mathrm{pH} 7.4)$ were passed during 10 minutes followed by an injection of PBS $\mathrm{pH} 7.4$ as running buffer and, to remove the bound SLN from the sensor surfaces, 1-minute regeneration steps with Triton X-100 1\%. The SPR signal assigned to each SLN solution corresponded to the plateau of the SPR response in the time in between the end of the SLN injection and the start of the regeneration step. Experiments were performed in triplicate.

\section{Results and Discussion}

3.1. Synthesis and Characterization of the SLN. SLN of different chemical composition with a common lipidic core composed of cetyl esters mixture and dispersed in poloxamer 188 as emulsifying agent were prepared by sonication method and, in some cases, modified by polymer coatings with PVA or chitosans. The mean diameter, zeta potential, and polydispersity index of the prepared SLN formulations were measured in aqueous solution and in PBS $1 \mathrm{x}$ as diluting medium (Tables 2(a) and 2(b), resp.). The particle size of the SLN ranges between 140 and $200 \mathrm{~nm}$, being comparable to the mean diameter measured in water or PBS in the case of P188+, $\mathrm{CL}$, and CM. An increase in the particle size when the SLN is measured in PBS is observed in P188 and PVA, probably due to the change in the hydrodynamic diameter of these nanovehicles in the high ionic strength medium. The polydispersity index of the obtained nanoparticles ranged between 0.14 and 0.23 , verifying in all cases the small polydispersity of the SLN solutions. It is well known that size influences both cell uptake and drug release kinetics in nanoparticles and other particulate systems [31-33]. Accordingly, it is desirable to attain low polydispersity since this is expected to result in a more predictable and homogeneous behavior within the targeted biological system.

In relation to the surface charge of the formulations, P188, P188+, and PVA appear to be negatively charged in both media, which is an interesting feature to maintain their stability and avoid possible aggregations. On the other side, significant changes in zeta potential values for chitosancoated nanoparticles are observed by changing deionized water for PBS in PCS measurement. Both CL and CM are characterized by positive zeta potentials in deionized water, in accordance with the positive surface charge expected from the chemical polymer structure of the coating. However, determination of zeta potential in PBS reflects a shift to more neutral values, which can be explained considering the 
TABLE 2

(a) Mean diameter ( $Z$-average), polydispersity index (PI), and zeta potential $(\zeta)$ of different formulations of solid lipid nanoparticles measured in deionized water. Data shown as mean \pm standard deviation, $n=3$.

\begin{tabular}{lccc}
\hline Sample & Mean diameter $(\mathrm{nm})$ & PDI & $\zeta(\mathrm{mV})$ \\
\hline SLN P188 & $199 \pm 3$ & $0.10 \pm 0.03$ & $-6.4 \pm 0.9$ \\
SLN PVA & $161 \pm 1$ & $0.213 \pm 0.006$ & $-1.5 \pm 0.1$ \\
SLN CM & $154 \pm 3$ & $0.183 \pm 0.002$ & $22 \pm 1$ \\
SLN CL & $151 \pm 3$ & $0.18 \pm 0.02$ & $13 \pm 2$ \\
SLN P188+ & $142 \pm 1$ & $0.154 \pm 0.008$ & $-1.1 \pm 0.1$ \\
\hline
\end{tabular}

(b) Mean diameter ( $Z$-average), polydispersity index (PI), and zeta potential $(\zeta)$ of different formulations of solid lipid nanoparticles measured in PBS as diluting medium. Data shown as mean \pm standard deviation, $n=3$.

\begin{tabular}{lccc}
\hline Sample & Mean diameter $(\mathrm{nm})$ & PDI & $\zeta(\mathrm{mV})$ \\
\hline SLN P188 & $207 \pm 2$ & $0.16 \pm 0.02$ & $-2.8 \pm 0.5$ \\
SLN PVA & $174 \pm 1$ & $0.237 \pm 0.009$ & $-6.1 \pm 0.1$ \\
SLN CM & $152.3 \pm 0.3$ & $0.175 \pm 0.007$ & $0.7 \pm 0.5$ \\
SLN CL & $146.5 \pm 0.8$ & $0.16 \pm 0.01$ & $2.2 \pm 0.2$ \\
SLN P188+ & $142 \pm 1$ & $0.16 \pm 0.01$ & $-5.2 \pm 0.4$ \\
\hline
\end{tabular}

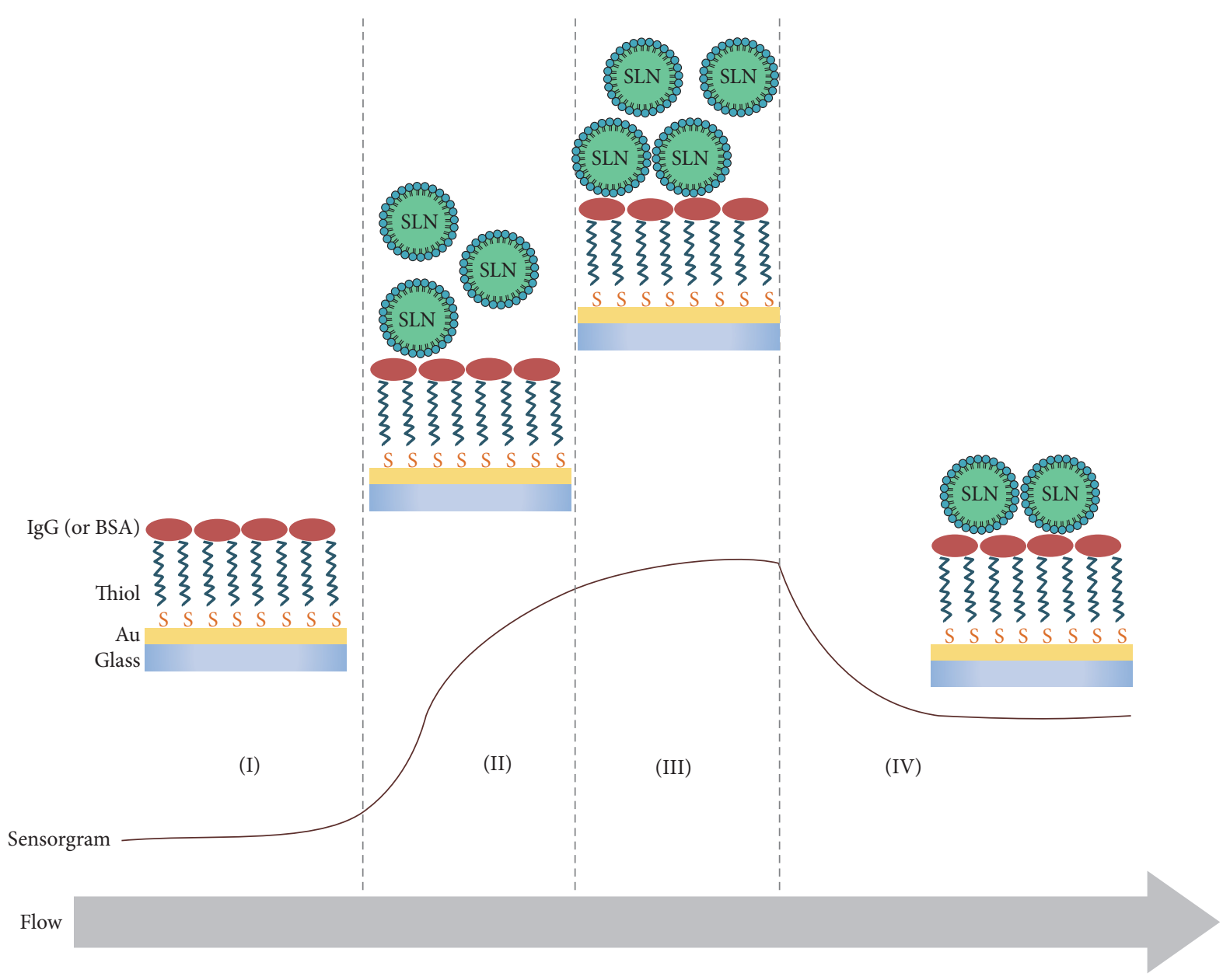

FIGURE 1: Simplified representation of the sensor surface to demonstrate the distinct layers of interest in the sensor surface and the correlation of the interactions that occurs in the sensor surface and the observed sensorgram. Roman numerals indicate (I) the protein layer after immobilization onto the thiol self-assembled monolayer, (II and III) SLN interaction with immobilized proteins at different times of the flow of SLN solutions, and (IV) the interaction of SLN-protein after restoration of buffer flow. 


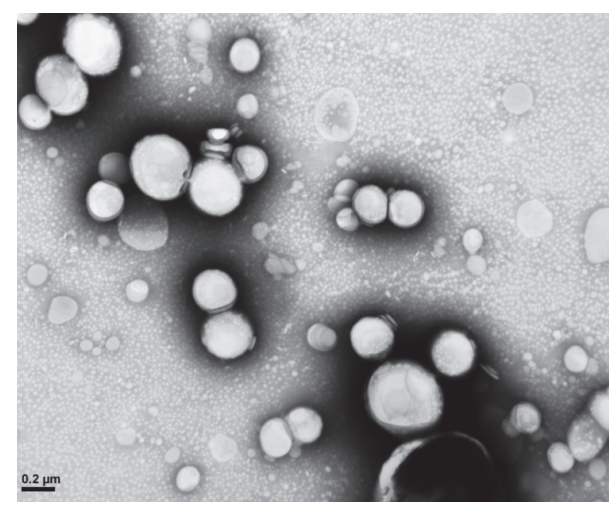

(a)

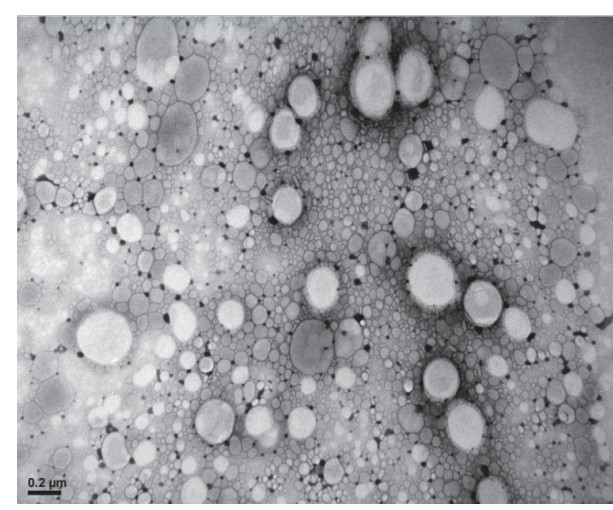

(b)

FIGURE 2: TEM images $(60,000 \mathrm{x})$ showing the effect of increasing surfactant concentration on SLN preparation: (a) SLN P188 and (b) SLN $\mathrm{P} 188+(\mathrm{b})$.

interaction between the positive surface charges of chitosan with negatively charged phosphate ions [34]. This interaction masks the positive charge of chitosan-coated SLN and produces particles with zeta potential values close to neutrality.

More information about the size, distribution, and morphology of SLN was obtained by TEM observations. An effect of increasing $50 \%$ of the surfactant concentration during SLN preparation was observed (Figure 2). While SLN P188 formulation exhibited nanoparticles around $200 \mathrm{~nm}$ in a relative narrow size distribution, the SLN P188+ preparation produced a bimodal mixture with similar nanoparticles of $200 \mathrm{~nm}$ in diameter but with the additional presence of small micelles (mean diameter $\leq 50 \mathrm{~nm}$ ), possibly due to the surfactant excess. This result is consistent with PCS measure, which indicated that the increase in surfactant concentration produces a lower mean diameter and higher PDI of nanoparticles.

On the other side, the effect of biopolymers addition during SLN preparation was also studied by TEM (Figure 3). The SLN PVA were characterized by nanoparticles in the range of $100-200 \mathrm{~nm}$ and covered by PVA layers. Interconnected nanoparticles maybe by PVA bridges are observed (Figures 3(a) and 3(b)). PCS experiments showed that PVA formulation exhibits mean diameters of $161 \pm$ $1 \mathrm{~nm}$ in water and $174 \pm 1 \mathrm{~nm}$ in PBS indicating that these bridges are consequence of the dryness process in the TEM chamber. The morphology, size, and distribution of chitosan nanoparticles seem not to be modified by changes in the MW of the biopolymer. In this sense, spherical SLN with a mean diameter around $200 \mathrm{~nm}$ were observed (Figures 3(c)-3(f)).

3.2. DLS Measurements of SLN-Protein Corona Complexes. In order to investigate the change in the nanoparticle size due to the adsorption of serum proteins, DLS measurements of SLN formulations incubated with Fetal Bovine Serum (FBS), as a model system of biological fluid, were performed (see Figure S1 in Supplementary Material available online at https:/doi.org/10.1155/2017/6509184). In all cases, the same increment of the particle size is observed (around $100 \mathrm{~nm}$ ) when the formulations are in contact with FBS, a finding that can be ascribed to the adsorption of serum proteins over the SLN independently of the differences in surface charge, chemical composition, and molecular weight of the coating in all tested SLN formulations.

The identity of the protein corona is known to play a fundamental role in biological adhesion [35], but due to the complexity of its composition, we propose to study the interaction of the nanovehicles with specific corona proteins like BSA and IgG. Due to the well-known ability of SPR technique to study biomolecular interactions, next we will discuss experimental results involving the immobilization of the proteins on the sensor surface and flowing SLN through the microfluidic system.

3.3. SPR Study of SLN Interaction with Immobilized IgG and $B S A$. Typical SPR sensorgrams resulting from the passage of SLN solutions over immobilized IgG and BSA are shown in Figures 4 and 5, respectively. In the figures, the SPR signal corresponding to each formulation is indicated in red. The SPR signal corresponding to the interaction of positively charged chitosan-coated nanoparticles with IgG or BSA, showed in (d) and (e) of Figures 4 and 5, is more intense in comparison with the SPR signal resulting from the other SLN. Even more, the binding of the positively charged chitosancoated formulations to the immobilized proteins cannot be regenerated with Triton X-100. However, chemical treatment with the nonionic surfactant allows recovering the sensor surface in the case of the other SLN. It is interesting to notice that this finding is independent of the chemical nature of the protein immobilized on the sensor surface, suggesting that the interaction of the SLN with proteins depends strongly on the physicochemical surface properties of the polymer coating. Considering that both immobilized proteins, BSA (isoelectric point $\simeq 4.7$ ) and $\operatorname{IgG}$ (isoelectric point $\simeq 7.0$ ), are negatively charged at the working $\mathrm{pH}$ of 7.4 and taking into account the fact that non-chitosan-coated SLN are also negatively charged, it could be inferred that electrostatic forces would not play an important role in the SLN-protein interactions. 


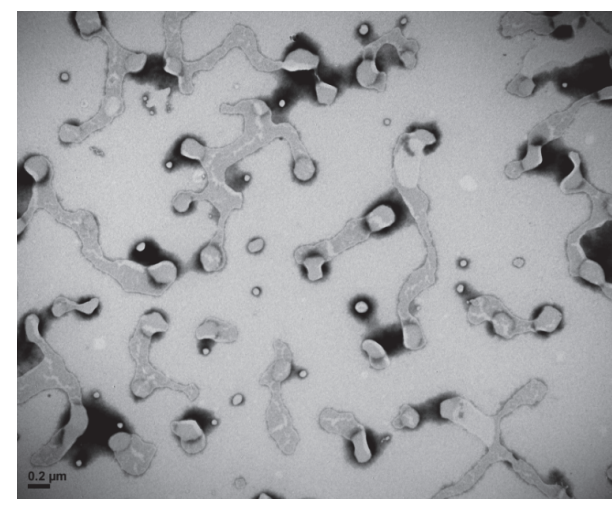

(a)

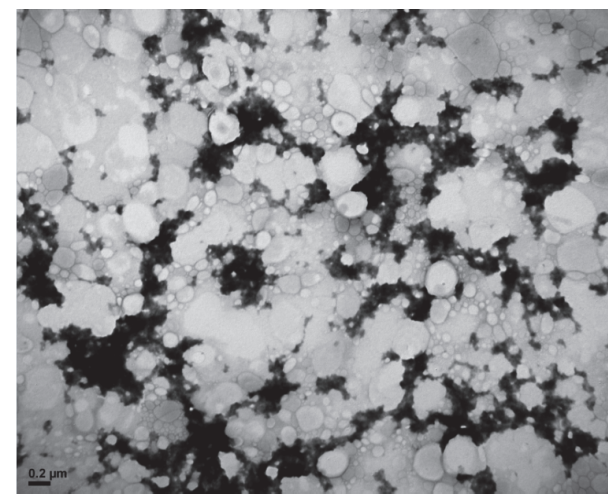

(c)

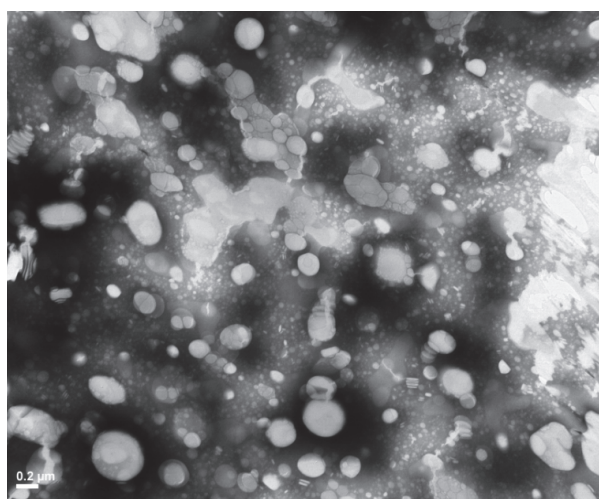

(e)

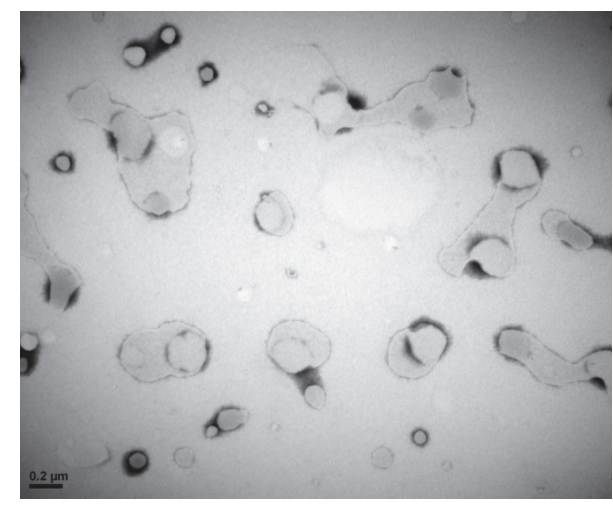

(b)

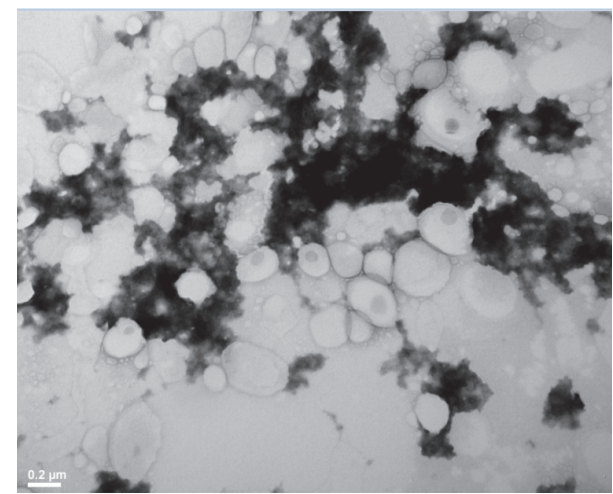

(d)

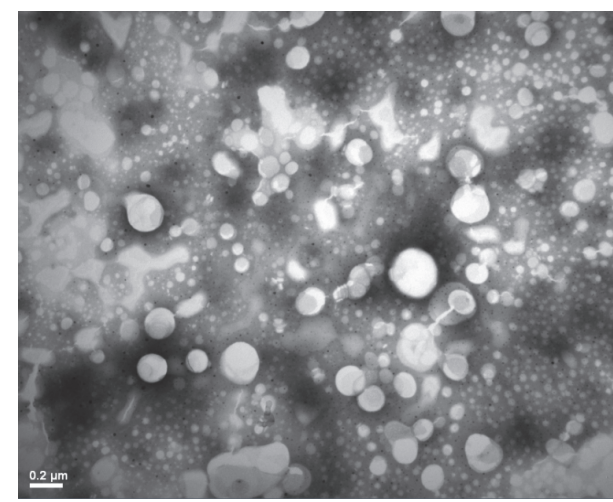

(f)

FIGURE 3: TEM images showing the effect on SLN preparation by addition of polymers observed by TEM: SLN PVA (a-b), SLN QL (c-d), and SLN QM (e-f) at 40,000 (a, c, and e) and 60,000 (b, d, f) magnifications.

Control experiments, flowing SLN over reference surfaces without immobilized proteins, showed negligible interaction in the case of P188, P188+, and PVA formulations (Figure 6). However, the chitosan-coated SLN showed roughly the same SPR signal change in the control experiments and in the measurements involving immobilized proteins. It is well known that due to its chemical nature, chitosan exhibits interactions with several surfaces and strong mucoadhesivity [36-39]. Thus, the interaction of chitosan-coated SLN with the surface in control experiments can be related to the interaction between the hydrophilic surface of the ester formed when MUA was subjected to carbodiimide coupling and the positively charged hydrophilic CL and CM. Another possibility is that in case that some carboxyl activated MUA terminal groups were not blocked by ethanolamine they can react with the nonprotonated amine groups of chitosan. It is well known that amines and hydroxyl groups situated on chitosan chains are responsible for chemical cross-linking. Hydrogen bonding can also contribute to the affinity of CL and CM with the MUA blocked surface.

In order to investigate if P188, P188+, and PVA formulations show preference to interact with IgG or with BSA, it is necessary to make a correction of the SPR signal that gives account of the amount of immobilized protein in each 


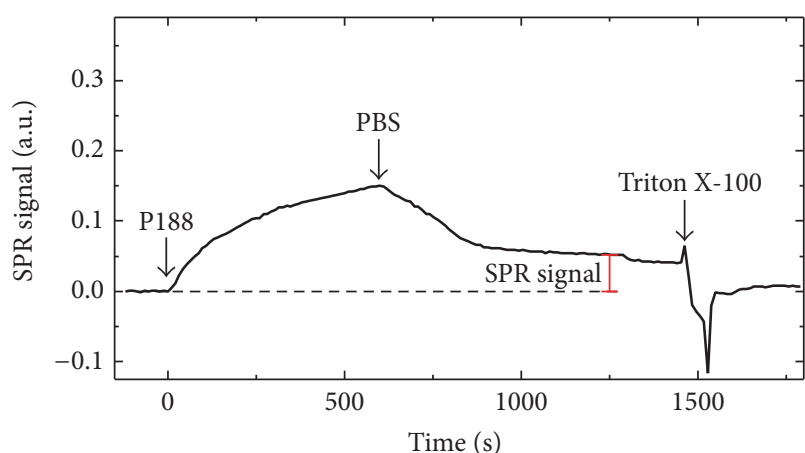

(a)

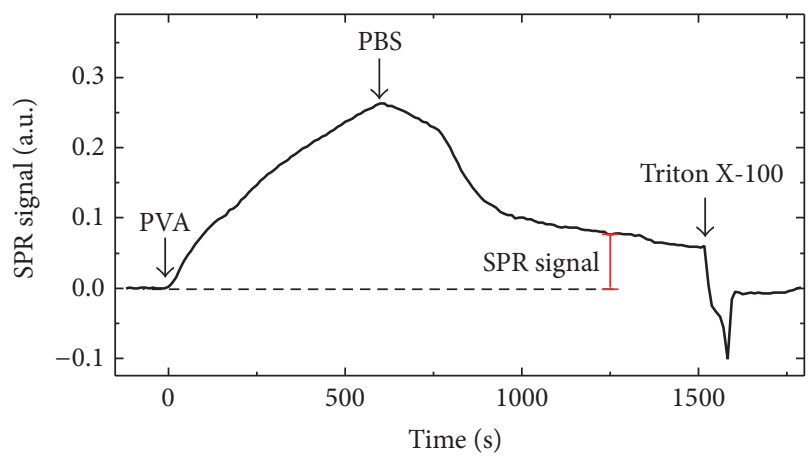

(c)

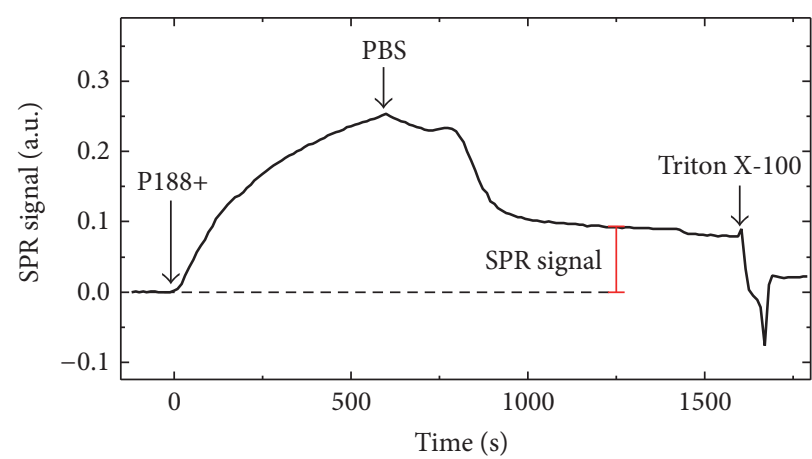

(b)

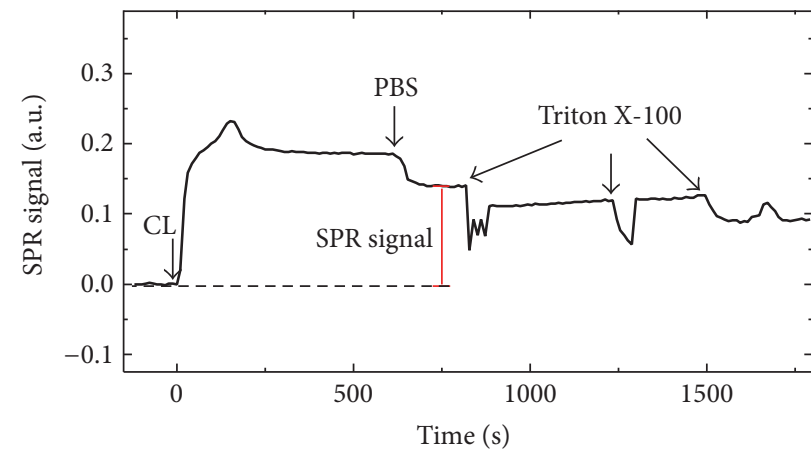

(d)

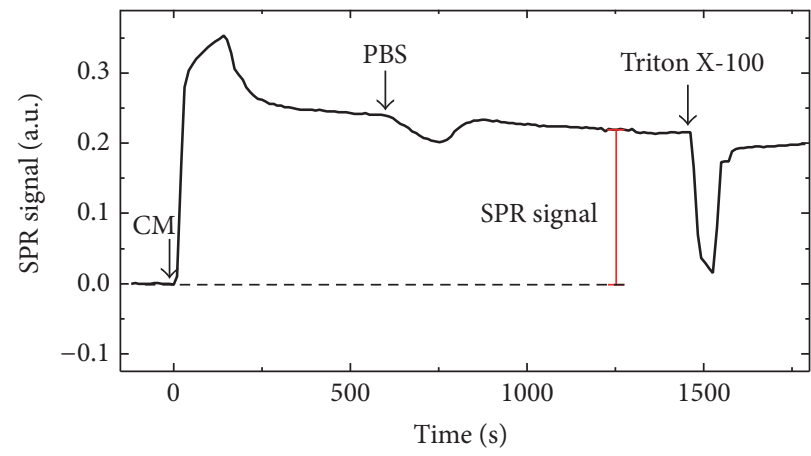

(e)

FIGURE 4: Sensorgrams showing the SPR signal resulting from the injection of the solid lipid nanoparticles (a) P188, (b) P188+, (c) PVA, (d) CL, and (e) CM over immobilized IgG (sensor surface: Au-MUA-IgG). Each cycle included a ten-minute injection of the SLN solution followed by a washing step with running buffer (PBS pH 7.4). In order to remove the bound SLN from the sensor surfaces, 1-minute regeneration steps with Triton X-100 were performed. SPR signal corresponding to each SLN was measured in a time in between the end of the SLN injection and the start of the regeneration step (around $1250 \mathrm{~s}$ of each cycle, except CL nanoparticles that the SPR signal was measured at around $750 \mathrm{~s})$.

experiment (Figure S2 in Supplementary Information). The corrected SPR signals corresponding to the interactions of the SLN and IgG or BSA are shown in Table 3. As it can be observed, all assayed nanosystems show preference to interact with IgG rather than BSA. Due to the fact that at $\mathrm{pH}$ 7.4 the surface charge of IgG is close to neutrality, another type of interactions excluding electrostatics should be explored to account for the experimental results.

Further studies are being carried out to validate the use of SLN formulations composed of P188, P188+, and PVA for their use in animal models in a next experimental step.

\section{Conclusions}

Solid lipid nanoparticles ranging 140-200 nm were obtained by the ultrasonication method. Distinct surface charges characterized the formulations, according to the chemical coating. In all cases a small polydispersity of the SLN solutions could be verified by photon correlation spectroscopy measurements. An increment of around $100 \mathrm{~nm}$ in the particle diameter was observed when the SLN formulations are incubated in FBS, finding that it can be ascribed to protein adsorption around the nanoparticles. 


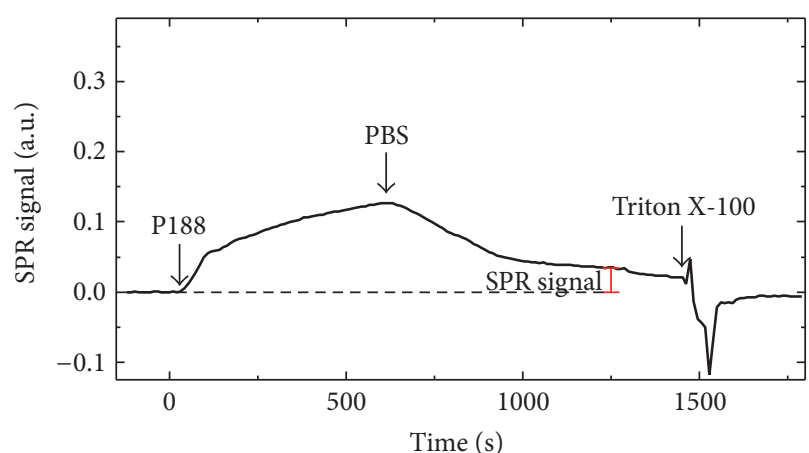

(a)

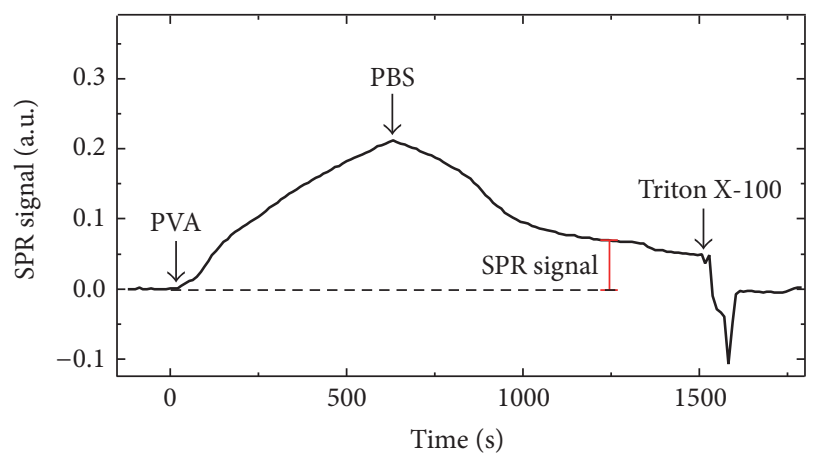

(c)

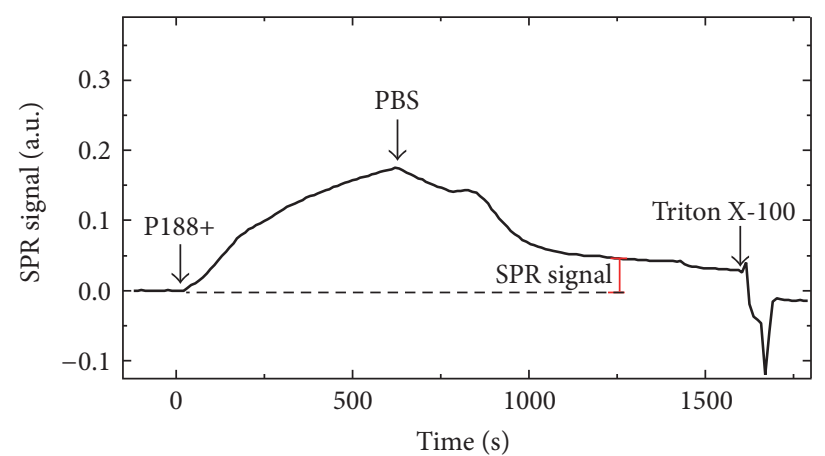

(b)

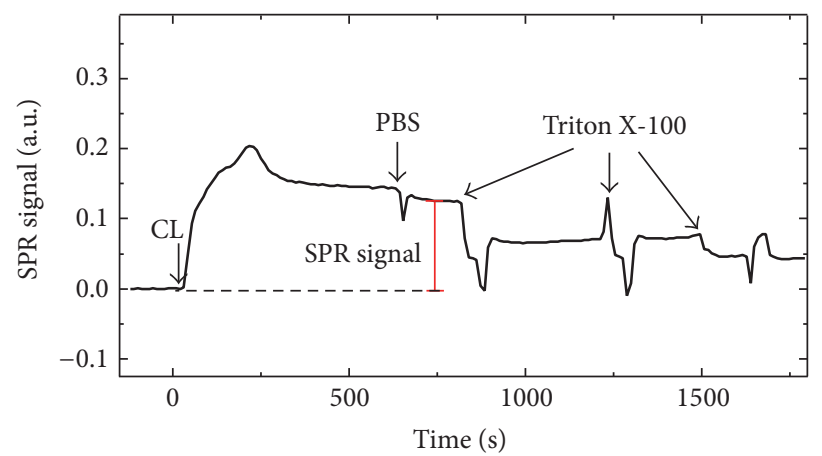

(d)

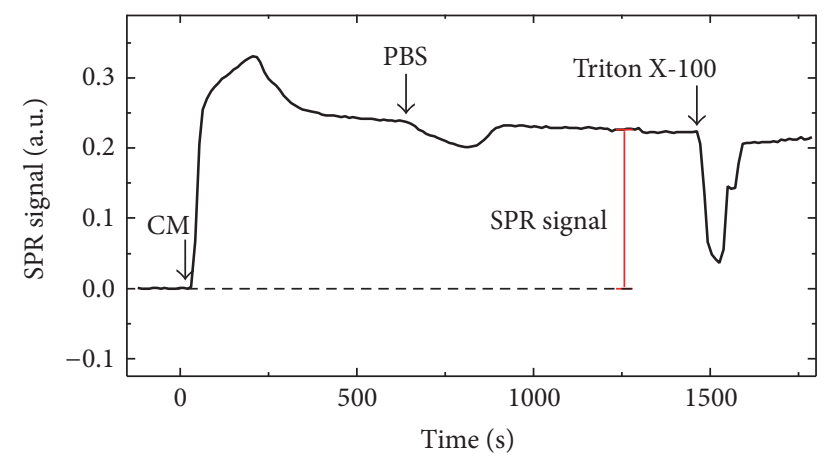

(e)

FIGURE 5: Sensorgrams showing the SPR signal resulting from the injection of the solid lipid nanoparticles (a) P188, (b) P188+, (c) PVA, (d) CL, and (e) CM over immobilized BSA (sensor surface: Au-MUA-BSA). Each cycle included a ten-minute injection of the SLN solution followed by a washing step with running buffer (PBS $\mathrm{pH}$ 7.4). In order to remove the bound SLN from the sensor surfaces, 1 min regeneration steps with Triton X-100 were performed. SPR signal corresponding to each SLN was measured in a time in between the end of the SLN injection and the start of the regeneration step (around $\sim 1250 \mathrm{~s}$ of each cycle, except CL nanoparticles that the SPR signal was measured at around 750 s).

The SPR experiments performed in this work verified different proclivity of non-chitosan-coated SLN to interact with IgG with respect to BSA, a fact that can be related to different circulations times in the bloodstream. Due to the complexity and the relative abundance of proteins of the plasma, this hypothesis should be confirmed in future in vivo studies. In the case of chitosan-coated SLN, the proposed strategy did not yield results that could gain information about the affinity of the nanoparticles and the studied proteins. However, the finding that these nanoparticles strongly interact with the sensor surface is in accordance with the fact that chitosan shows affinity with a variety of surfaces.
We demonstrate that SPR is a suitable technique to evaluate the interactions between blood proteins and nonchitosan-coated SLN. In these cases it is possible to obtain information about the different relative affinities of the formulations and the studied proteins, by correcting the SPR signal change for the amount of immobilized protein in the sensor surface. The approach discussed in this paper can be extended to study the interactions of non-chitosancoated SLN with other components of the protein corona like proteins of the complement pathway and fibrinogen. Therefore, the application of SPR can be considered as a promising tool for the evaluation of SLN-protein interactions 
TABLE 3: Normalized SPR signal for each SLN formulation (for more detail concerning the calculation see Figure S2 in Supplementary Information). * Ratio between the normalized SPR signal for immobilized IgG and the normalized SPR signal for immobilized BSA.

\begin{tabular}{lccc}
\hline & \multicolumn{3}{c}{ Normalized SPR signal } \\
Sample & Au-MUA-IgG & Sensor surface & Au-MUA-BSA \\
P188 & 0.78 & 0.13 & 6.00 \\
P188+ & 1.71 & 0.17 & 10.05 \\
PVA & 1.44 & 0.20 & 7.20 \\
\hline
\end{tabular}

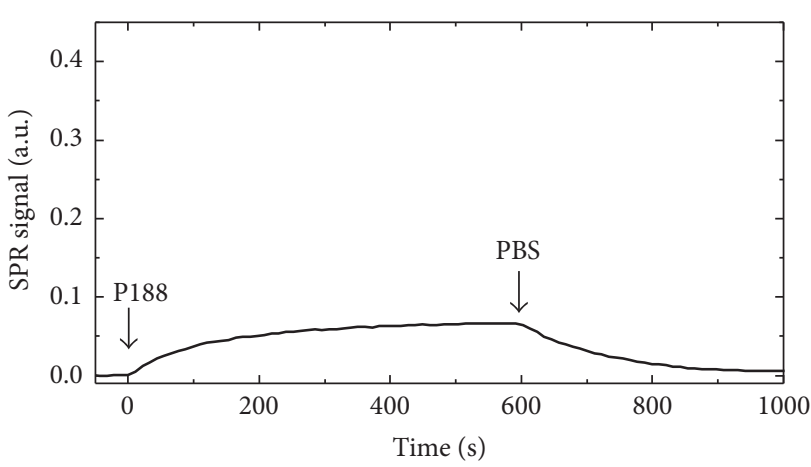

(a)

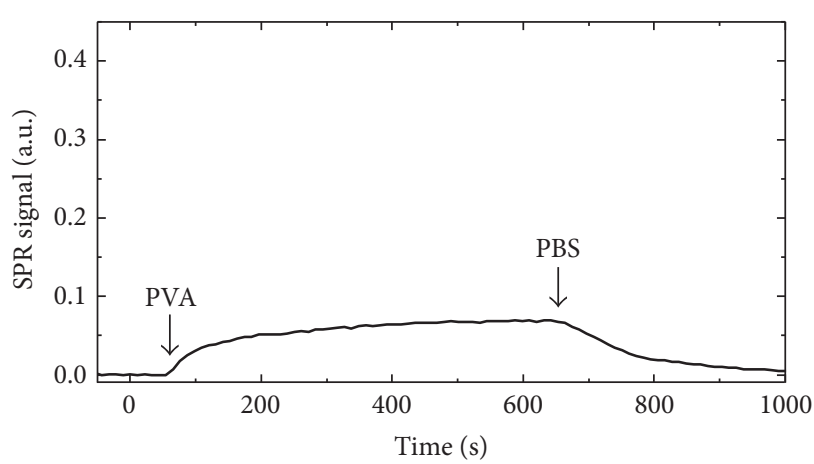

(c)

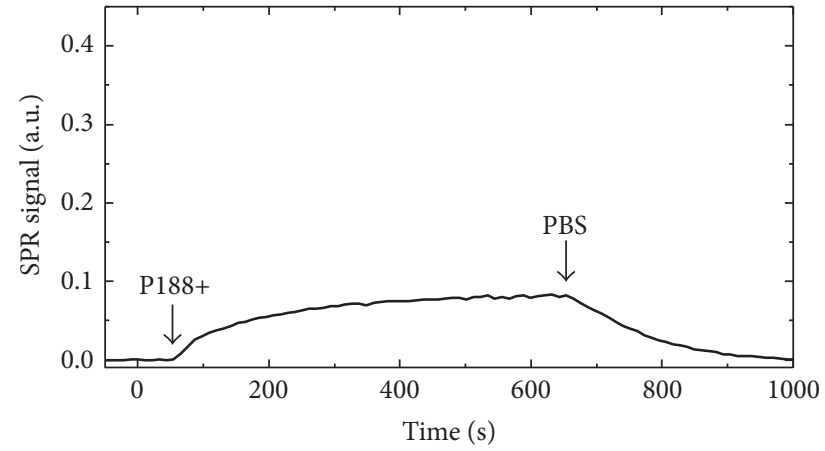

(b)

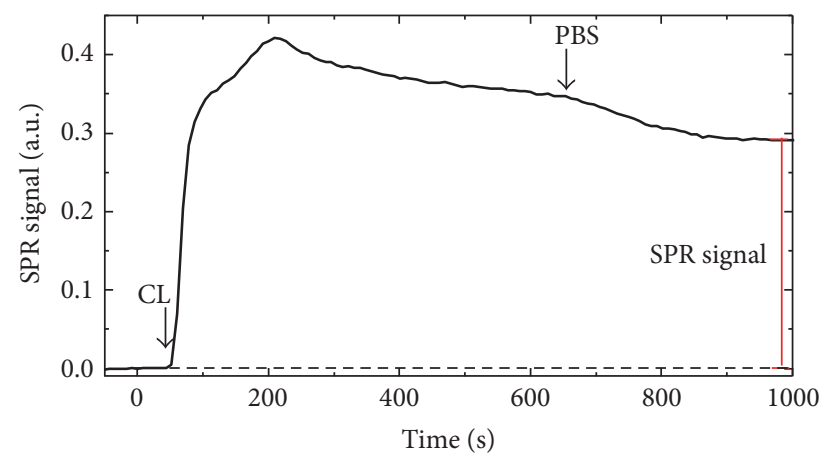

(d)

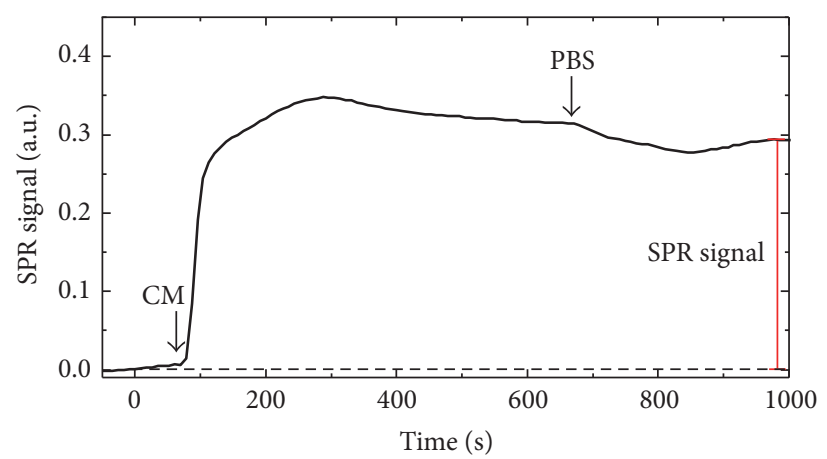

(e)

FIGURE 6: Sensorgrams showing the SPR signal resulting from the injection of the solid lipid nanoparticles (a) P188, (b) P188+, (c) PVA, (d) $\mathrm{CL}$, and (e) CM over control surface without immobilized protein (sensor surface: Au-MUA). Each cycle included a ten-minute injection of the SLN solution followed by a washing step with running buffer (PBS pH 7.4). SPR response arising from the interaction of chitosan-coated SLN and the control surface corresponds to the stabilization of the signal after the end of the SLN injection (at around $1000 \mathrm{~s}$ ). 
in vitro, as well as for predicting the blood clearance of liposomes and likely other nanocarriers in vivo.

\section{Conflicts of Interest}

The authors declare that they have no conflicts of interest regarding the publication of this paper.

\section{Acknowledgments}

Mauricio E. Di Ianni is a postdoctoral fellow of CONICET. Germán A. Islan, Cecilia Y. Chain, Guillermo R. Castro, and Alan Talevi are members of the research career of CONICET. María E. Vela is member of the research career of CIC PBA. The authors thank CONICET and INTA for support through Grants PIP 0671 and PNNAT-1128043.

\section{References}

[1] E. Luque-Michel, E. Imbuluzqueta, V. Sebastián, and M. J. Blanco-Prieto, "Clinical advances of nanocarrier-based cancer therapy and diagnostics," Expert Opinion on Drug Delivery, vol. 14, no. 1, pp. 75-92, 2017.

[2] D. Carradori, J. Eyer, P. Saulnier, V. Préat, and A. des Rieux, "The therapeutic contribution of nanomedicine to treat neurodegenerative diseases via neural stem cell differentiation," Biomaterials, vol. 123, pp. 77-91, 2017.

[3] P. L. Lam, W. Y. Wong, Z. Bian, C. H. Chui, and R. Gambari, "Recent advances in green nanoparticulate systems for drug delivery: Efficient delivery and safety concern," Nanomedicine, vol. 12, no. 4, pp. 357-385, 2017.

[4] M. Moritz and M. Geszke-Moritz, "Recent developments - In the application of polymeric nanoparticles as drug carriers," Advances in Clinical and Experimental Medicine, vol. 24, no. 5, pp. 749-758, 2015.

[5] A. Vedda and I. Villa I, "Medical applications of nanomaterials," in Nano-Optics: Principles Enabling Basic Research and Applications, B. Di Bartolo, J. Collins, and L. Silvestri, Eds., NATO Science for Peace and Security Series B: Physics and Biophysics, pp. 369-386, Springer, Dordrecht, Netherlands, 2017.

[6] P. Foroozandeh and A. A. Aziz, "Merging Worlds of Nanomaterials and Biological Environment: Factors Governing Protein Corona Formation on Nanoparticles and Its Biological Consequences," Nanoscale Research Letters, vol. 10, no. 1, article no. 221, 2015.

[7] G. Caracciolo, O. C. Farokhzad, and M. Mahmoudi, "Biological Identity of Nanoparticles In Vivo: Clinical Implications of the Protein Corona," Trends in Biotechnology, 2016.

[8] H. Gao and Q. He, "The interaction of nanoparticles with plasma proteins and the consequent influence on nanoparticles behavior," Expert Opinion on Drug Delivery, vol. 11, no. 3, pp. 409-420, 2014.

[9] C. Gunawan, M. Lim, C. P. Marquis, and R. Amal, "Nanoparticle-protein corona complexes govern the biological fates and functions of nanoparticles," Journal of Materials Chemistry B, vol. 2, no. 15, pp. 2060-2083, 2014.

[10] J. Lazarovits, Y. Y. Chen, E. A. Sykes, and W. C. W. Chan, "Nanoparticle-blood interactions: the implications on solid tumour targeting," Chemical Communications, vol. 51, no. 14, pp. 2756-2767, 2015.
[11] P. Aggarwal, J. B. Hall, C. B. McLeland, M. A. Dobrovolskaia, and S. E. McNeil, "Nanoparticle interaction with plasma proteins as it relates to particle biodistribution, biocompatibility and therapeutic efficacy," Advanced Drug Delivery Reviews, vol. 61, no. 6, pp. 428-437, 2009.

[12] J. S. Suk, Q. Xu, N. Kim, J. Hanes, and L. M. Ensign, "PEGylation as a strategy for improving nanoparticle-based drug and gene delivery," Advanced Drug Delivery Reviews, vol. 99, pp. 28-51, 2016.

[13] G. D. Mogoşanu, A. M. Grumezescu, C. Bejenaru, and L. E. Bejenaru, "Polymeric protective agents for nanoparticles in drug delivery and targeting," International Journal of Pharmaceutics, vol. 510, no. 2, pp. 419-429, 2016.

[14] A. Anselmo and S. Mitragotri, "Nanoparticles in the Clinic," Bioengineering \& Translational Medicine, vol. 1, no. 1, 2016.

[15] B. Rodenak-Kladniew, G. Islan, M. de Bravo, N. Durán, G. Castro, and G. R. Design, "Characterization and in vitro evaluation of linalool-loaded solid lipid nanoparticles as potent tool in cancer therapy," Colloids and Surfaces B: Biointerfaces, vol. 154, 2017.

[16] W. Mehnert and K. Mäder, "Solid lipid nanoparticles: production, characterization and applications," Advanced Drug Delivery Reviews, vol. 64, pp. 83-101, 2012.

[17] C. Schwarz and W. Mehnert, "Freeze-drying of drug-free and drug-loaded solid lipid nanoparticles (SLN)," International Journal of Pharmaceutics, vol. 157, no. 2, pp. 171-179, 1997.

[18] J. Homola, "Surface Plasmon Resonance Based Sensors," in Methods and Applications, O. S. Wolfbei, Ed., vol. 4 of Springer Series on Chemical Sensors and Biosensors, pp. 45-67, SpringerVerlag, Berlin, Heidelberg, Germany, 2006.

[19] N. J. de Mol and M. J. Fischer, Surface Plasmon Resonance Methods and Protocols, Springer, London, 2010.

[20] X. Guo, "Surface plasmon resonance based biosensor technique: a review," Journal of Biophotonics, vol. 5, no. 7, pp. 483-501, 2012.

[21] H. H. Nguyen, J. Park, S. Kang, and M. Kim, "Surface plasmon resonance: a versatile technique for biosensor applications," Sensors (Switzerland), vol. 15, no. 5, pp. 10481-10510, 2015.

[22] M. Canovi, J. Lucchetti, M. Stravalaci et al., "Applications of Surface Plasmon Resonance (SPR) for the characterization of nanoparticles developed for biomedical purposes," Sensors (Switzerland), vol. 12, no. 12, pp. 16420-16432, 2012.

[23] O. K. Kari, T. Rojalin, S. Salmaso et al., "Multi-parametric surface plasmon resonance platform for studying liposome-serum interactions and protein corona formation," Drug Delivery and Translational Research, vol. 7, no. 2, pp. 228-240, 2017.

[24] B. J. Crielaard, A. Yousefi, J. P. Schillemans et al., "An in vitro assay based on surface plasmon resonance to predict the in vivo circulation kinetics of liposomes," Journal of Controlled Release, vol. 156, no. 3, pp. 307-314, 2011.

[25] L. Morelli, L. Plotkin, J. Leoni, C. A. Fossati, and R. A. Margni, "Analysis of oligosaccharides involved in the asymmetrical glycosylation of igg monoclonal antibodies," Molecular Immunology, vol. 30, no. 7, pp. 695-700, 1993.

[26] V. Venkateswarlu and K. Manjunath, "Preparation, characterization and in-vitro release kinetics of clozapine solid lipid nanoparticles," Journal of Controlled Release, vol. 95, no. 3, pp. 627-638, 2004.

[27] G. Islan, P. Tornello, G. Abraham, N. Duran, and G. Castro, "Smart lipid nanoparticles containing levofloxacin and DNase for lung delivery. Design and characterization," Colloids and Surfaces B: Biointerfaces, vol. 143, pp. 168-176, 2016. 
[28] R. M. Shah, F. Malherbe, D. Eldridge, E. A. Palombo, and I. H. Harding, "Physicochemical characterization of solid lipid nanoparticles (SLNs) prepared by a novel microemulsion technique," Journal of Colloid and Interface Science, vol. 428, pp. 286-294, 2014.

[29] D. Pozzi, G. Caracciolo, L. Digiacomo et al., "The biomolecular corona of nanoparticles in circulating biological media," Nanoscale, vol. 7, no. 33, pp. 13958-13966, 2015.

[30] G. T. Hermanson, Bioconjugate Techniques, Academic Press, San Diego, California, 2008.

[31] M. Halayqa and U. Domańska, "PLGA biodegradable nanoparticles containing perphenazine or chlorpromazine hydrochloride: effect of formulation and release," International Journal of Molecular Sciences, vol. 15, no. 12, pp. 23909-23923, 2014.

[32] N. S. Berchane, K. H. Carson, A. C. Rice-Ficht, and M. J. Andrews, "Effect of mean diameter and polydispersity of PLG microspheres on drug release: Experiment and theory," International Journal of Pharmaceutics, vol. 337, no. 1-2, pp. 118126, 2007.

[33] M. Caldorera-Moore, N. Guimard, L. Shi, and K. Roy, "Designer nanoparticles: incorporating size, shape and triggered release into nanoscale drug carriers," Expert Opinion on Drug Delivery, vol. 7, no. 4, pp. 479-495, 2010.

[34] Y. Zhang and M. Zhang, "Calcium phosphate/chitosan composite scaffolds for controlled in vitro antibiotic drug release," Journal of Biomedical Materials Research, vol. 62, no. 3, pp. 378386, 2002.

[35] P. Decuzzi and M. Ferrari, "The role of specific and non-specific interactions in receptor-mediated endocytosis of nanoparticles," Biomaterials, vol. 28, no. 18, pp. 2915-2922, 2007.

[36] F. Ahmadi, Z. Oveisi, S. Mohammadi Samani, and Z. Amoozga, "Chitosan based hydrogels: characteristics and pharmaceutical applications," Research in Pharmaceutical Sciences, vol. 10, 2015.

[37] G. Z. Kyzas and D. N. Bikiaris, "Recent modifications of chitosan for adsorption applications: A critical and systematic review," Marine Drugs, vol. 13, no. 1, pp. 312-337, 2015.

[38] L. Bekale, D. Agudelo, and H. A. Tajmir-Riahi, "Effect of polymer molecular weight on chitosan-protein interaction," Colloids and Surfaces B: Biointerfaces, vol. 125, pp. 309-317, 2015.

[39] V. Boeris, B. Farruggia, and G. Picó, "Chitosan-bovine serum albumin complex formation: A model to design an enzyme isolation method by polyelectrolyte precipitation," Journal of Chromatography B: Analytical Technologies in the Biomedical and Life Sciences, vol. 878, no. 19, pp. 1543-1548, 2010. 

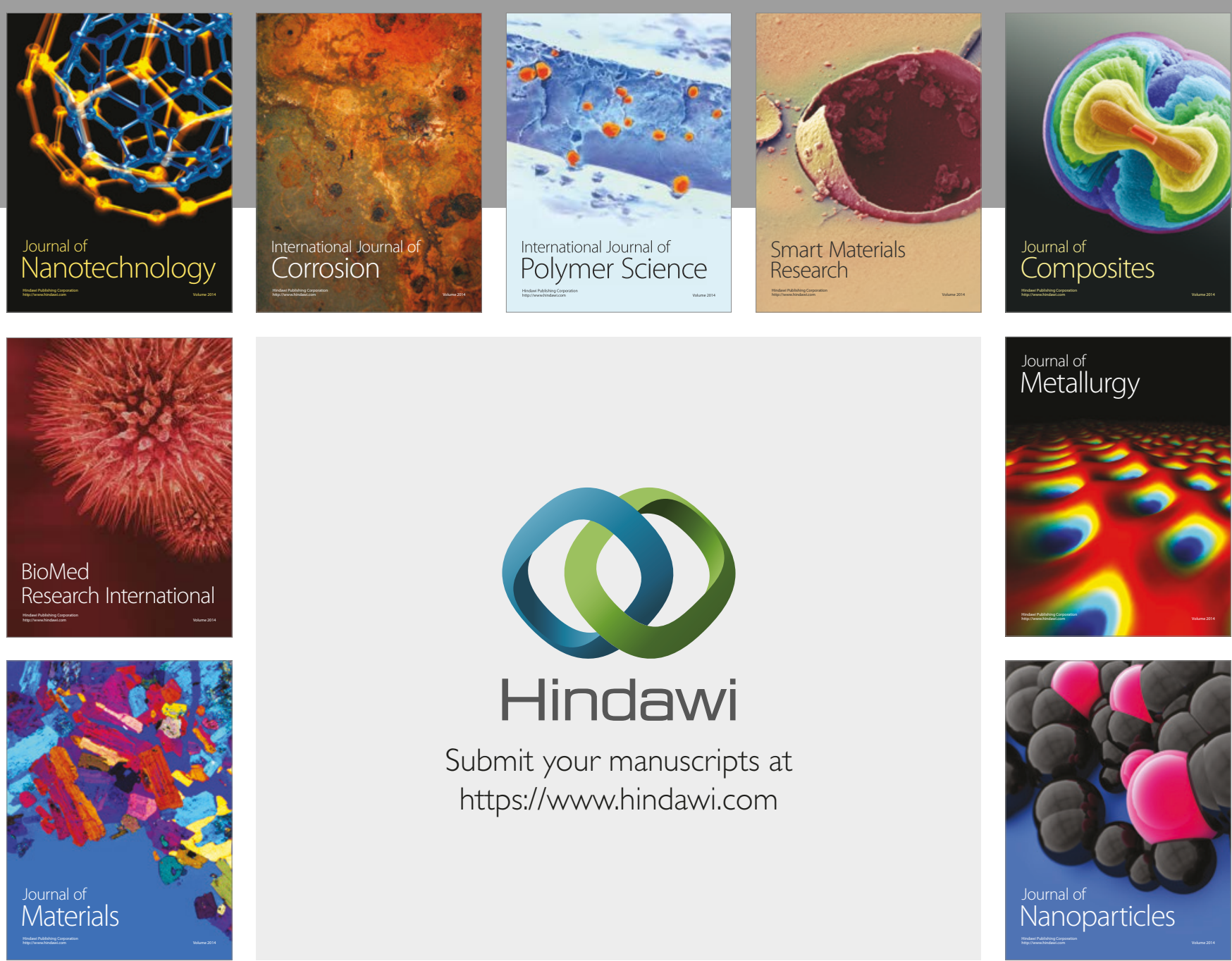

\section{Hindawi}

Submit your manuscripts at

https://www.hindawi.com
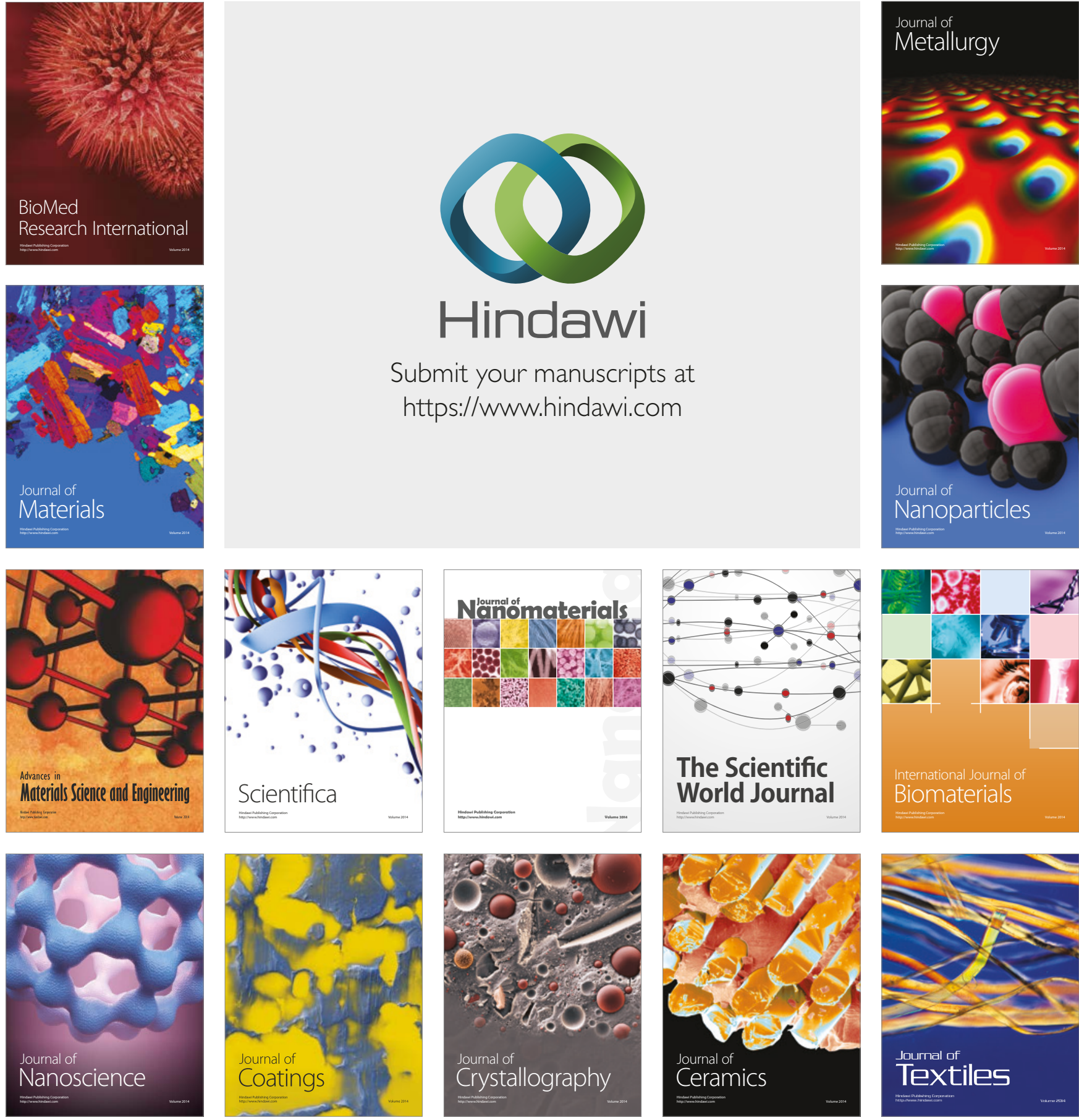

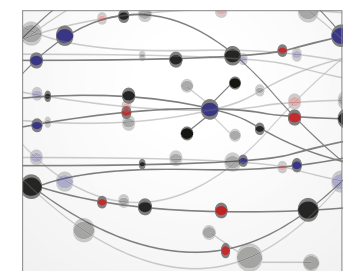

The Scientific World Journal
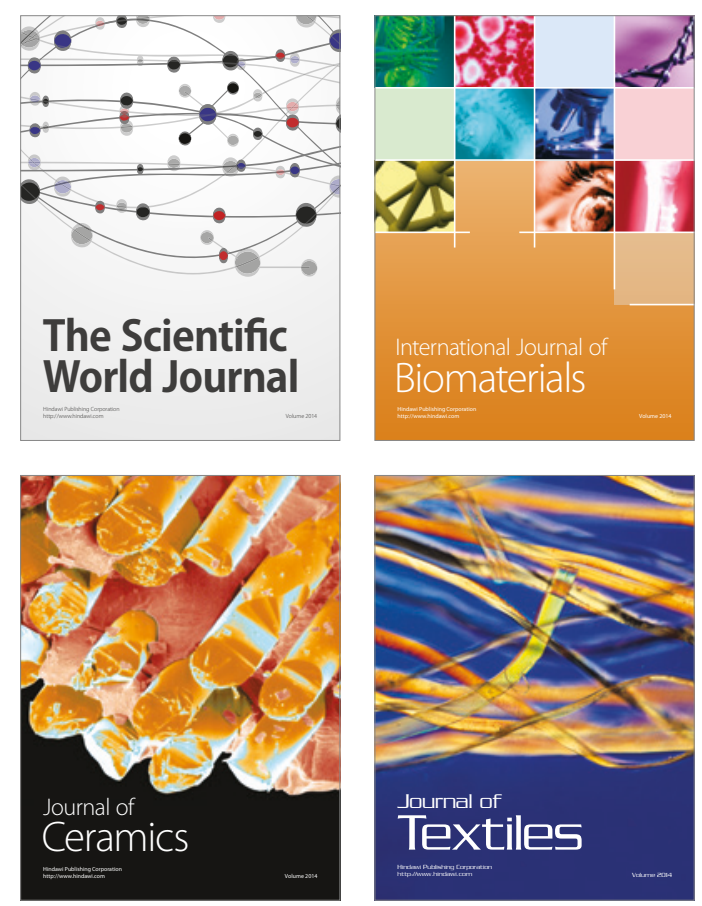